\title{
Neue Räume - neue Rollen? Ungewissheit im Kontext der Temporären Organisation
}

\author{
Martin Elbe $^{1}$ (D) S Sibylle Peters \\ Angenommen: 9. September 2021 / Online publiziert: 26. Oktober 2021 \\ ๑) Springer Fachmedien Wiesbaden GmbH, ein Teil von Springer Nature 2021
}

\section{Zusammenfassung}

Dieser Beitrag in der Zeitschrift „Gruppe. Interaktion. Organisation. (GIO)“ diskutiert die Auswirkung von Ungewissheitserleben und neuen betrieblichen Handlungsräumen auf die Rollen- und Identitätskonstruktion von Mitarbeitern und Mitarbeiterinnen. Ungewissheit ist nicht erst seit Auftreten der Corona-Pandemie eine der zentralen Herausforderungen hinsichtlich der Entwicklung von Arbeitsbedingungen in Organisationen. Digitalisierung und Virtualisierung, Wissensarbeit und Projektorganisation sind Trends in der Arbeitswelt, die fragen lassen, wie sich soziale Identität und Rollenverhalten in Temporären Organisationen unter dem Einfluss von Ungewissheit entwickeln. Hierzu wird im vorliegenden Artikel das Verhältnis von temporären Repräsentationsräumen und Identität sowie neuen Rollen in Temporären Organisationen, unter besonderer Berücksichtigung von Projektstrukturen, untersucht. Es wird deutlich, dass durch die räumliche Neupositionierung der Temporären Organisation und dem damit verbundenen Wandel der Mitarbeiterrollen der Identitätsarbeit auf Basis der eigenen Erwerbsbiographie eine immer größere Bedeutung zukommt.

Schlüsselwörter Identität · Employography $\cdot$ Projekte $\cdot$ Raumtheorie $\cdot$ Rollen $\cdot$ Temporäre Organisation $\cdot$ Ungewissheit

\section{New spaces-new roles? Uncertainty in the context of the temporary organization}

\begin{abstract}
This paper in the journal "Gruppe. Interaktion. Organisation. (GIO)" discusses the impact of uncertainty and new operational space on the role and identity construction of employees. Uncertainty has not only been one of the central challenges in terms of the development of working conditions and organizations since the emergence of the Corona pandemic. Digitization and virtualization, knowledge work and project organization are trends in the world of work that raise questions about how social identity and role behavior develop in temporary organizations under the influence of uncertainty. For this purpose, this article examines the relationship between temporary spatial representation and identity as well as new roles in temporary organizations, with special attention to project structures. It becomes clear due to that the spatial repositioning of the temporary organization and the associated change in the employee roles employography as an approach to identity work based on one's own employment biography is becoming increasingly important.
\end{abstract}

Keywords Identity $\cdot$ Employography $\cdot$ Projects $\cdot$ Roles $\cdot$ Temporary Organization $\cdot$ Theory of Space $\cdot$ Uncertainty

\section{Einleitung: Das Problem mit der Ungewissheit}

Tatsächlich: Mit dem Auftreten des Corona-Virus ist das Problem der Ungewissheit in der Mitte der Gesellschaft an-

Prof. Dr. Martin Elbe

martin.elbe@t-online.de

Zeppelinstr. 127/128, 14471 Potsdam, Deutschland gekommen. Jede und jeder ist betroffen - nicht zwangsläufig von einer Infektion durch den Virus selbst, wohl aber von den gesellschaftlichen und wirtschaftlichen Auswirkungen der Krankheit, die seit Ende 2019 von Südost-Asien aus 
die gesamte Welt verändern. Speziell hinsichtlich der Bewältigung von Krisen - wie der Pandemie, aber auch dem Klimawandel - gilt das Diktum der Katastrophensoziologie, dass es keine Naturkatastrophen gibt, sondern nur für Menschen als katastrophal erlebte Konsequenzen von Umweltereignissen (Clausen 2007, S. 94). Und genau im Psychosozialen müssen sich gruppendynamische Prozesse in Krisen und Katastrophen bewähren. Führende (als „Schutzexperten“) und alle Anderen (als „Schutzbedürftige“) sind in sozialen Systemen über eine gemeinsame Sinnkonstruktion aneinander gebunden (Clausen und Dombrovsky 1983; Elbe 2020). ${ }^{1}$ Sie sind sich einig darüber, dass die Elite, das Management, die Herrschenden wissen wie die Krise am besten zu bewältigen sei. Erst wenn es der Führungselite nicht mehr gelingt diesen gemeinsamen Sinnkontext (und verbunden damit das Vertrauen in ihre Führungsfähigkeit) herzustellen, wird die natürliche Bedrohung als unabwendbar wahrgenommen - als ein Schicksal dem der oder die Einzelne ohnmächtig gegenüber steht und dem man dann eben schutzlos ausgeliefert ist. Eine handlungsauslösende Zielantizipation innerhalb eines vertrauten Systems ist kaum mehr möglich und ebenso versagen streng regelgeleitete oder automatisierte Formen der Handlungswahl (vgl. zur Psychologie der Handlungswahl Hommel und Nattkemper 2011, S. 97ff). Dies impliziert aber, dass auch RisikoAbwägungen kaum mehr greifen können, da Wahrscheinlichkeiten von Handlungserfolgen nicht mehr abgewogen werden können. ${ }^{2}$

Wie Böhle und Busch (2012) zeigen, liegt hier aber auch die Chance von Ungewissheit: Es besteht die Möglichkeit Handlungsoptionen jenseits von Ohnmacht und Kontrolle $\mathrm{zu}$ finden und damit neue Sinnkontexte in einem System zu entdecken. Aufgrund des Mangels an Erfahrung haben bei Ungewissheit Intuition und Heuristiken eine höhere Erfolgswahrscheinlichkeit als andere Handlungsgrundlagen, da sie das Nichtwissen als Entscheidungsgrundlage mit einbeziehen - sie ermöglichen eine begründbare und damit auch lernorientierte Handlung (Gigerenzer 2007), die auf aktuellen, temporären und nicht auf grundsätzlichen, quasi verbrieften Erfolg abzielt. Erst die Wiederholung von Handlungssequenzen im Erfolgsfall und deren Verstetigung und Institutionalisierung begrenzen die Ungewissheit und damit zugleich die Offenheit des Erfolgsraumes. Die kalkulierte oder regelgebundene Handlung im Rahmen verfestigter Institutionen beruht aber nicht nur auf generellen Entwicklungsprozessen, sondern - und darauf machte Kurt Lewin

\footnotetext{
${ }^{1}$ Diese Forschungsrichtung stellt damit einen anderen Aspekt, als die Grenzproduktivität einer Gesellschaft (vgl. hierzu Tainter 1990), in das Zentrum der Betrachtung wie Gesellschaften mit Krisen und Katastrophen umgehen. Eine deterministische Sicht wird so zugunsten einer sinnvermittelnde Chancensuche vermieden.

2 Zum Umgang mit Risiko in der modernen Gesellschaft vgl. Beck (1986), Bonß (1995).
}

bereits 1947 (S. 35ff) aufmerksam - auf Gruppendynamik und realen Entscheidungsprozessen sozialer Gruppen. Diese Entscheidungen finde vor Ort in konkreten sozialen Settings (die Lewin als soziale Felder konzipiert) statt und die spezifische Punkte im Raum aller Handlungsmöglichkeiten eines dynamischen Entscheidungssystems (,phase space“ bei Lewin 1947, S. 38) kennzeichnen. Eine solche Entscheidung führt also im Falle einer (Wieder-) Stabilisierung des Systems zu einem temporären, quasi-stationärem Gleichgewicht der Handlungsabstimmung, das nun handlungsleitend wirkt und hilft Ungewissheit als gestaltbare Offenheit zu erleben.

Vor diesem Hintergrund wird im vorliegenden Artikel die Frage gestellt, wie sich soziale Identität und Rollenverhalten in Temporären Organisationen unter dem Einfluss von Ungewissheit entwickeln und ausdifferenzieren. Hierzu wird im folgenden 2. Kapitel das Verhältnis von temporären Repräsentationsräumen und Identität untersucht. Das 3. Kapitel analysiert neue Rollen in Temporären Organisationen, unter besonderer Berücksichtigung von Projektstrukturen. Das (abschließende) 4. Kapitel fasst die zentralen Ergebnisse zusammen. Der Beitrag stellt eine theoretische Analyse aktueller sozialer Entwicklungen in unserer Arbeitswelt und Gesellschaft ins Zentrum der Diskussion, stützt sich aber auch auf eigene empirische Befunde und Beispiele ab.

\section{Temporäre Repräsentationsräume und Identitäten}

\subsection{Ungewissheit und neuen Repräsentationsräume}

Ungewissheit ist ein Zustand, der in verschiedenen Disziplinen schon länger untersucht und diskutiert wird. Dabei kommen recht unterschiedliche Aspekte des Phänomens in den Blick: Ungewissheit kann als Raum gesehen werden, der die Entscheidungsfähigkeit des Menschen begründet (Wust 1940) oder als gesellschaftliches Problem der Moderne, das Risiken aufscheinen lässt (Bonß 1995). Ungewissheit kann pädagogische Felder in der Moderne aufzeigen (Helsper et al. 2003) oder Gegenstand psychologischer Diagnostik sein (Dalbert 2002). In all diesen Sichtweisen geht es um Selbstverortung und Sinnsuche. Ungewissheit kann damit als kontingenter Verweis des Ichs auf eine (sozial-) räumliche Umwelt verstanden werden, als Kern dessen, was in der Identitätsarbeit (Keupp und Höfer 1997) durch das Individuum immer wieder neu erzeugt wird. In der Moderne wird diese Selbstverortung zur Herausforderung, da die Geborgenheit im Vertrauten immer mehr zur Disposition gestellt wird.

Dies zeigte sich auch im Zuge der Entwicklung der Corona-Pandemie 2020/2021 in Deutschland. In der ersten Phase der Krankheitswelle im Frühjahr 2020 wurden Einschrän- 
kungen weitgehend akzeptiert - die Führungsfunktion der Eliten wurde von der breiten Masse nicht angezweifelt. In der zweiten Phase des Sommers 2020 schien das Krankheitsgeschehen abzuflauen, viele versuchten im Urlaub, zuhause oder sogar im Ausland, ein Gefühl von Normalität der Ungewissheitserfahrung des Frühjahrs entgegen zu stellen. Dies konnte aber nicht für alle gelten - so blieben Massenveranstaltungen untersagt und damit zahlreiche Unternehmer und Organisationen von einer Normalisierung ausgeschlossen. In anderen Organisationen wurde das Arbeiten im Home-Office in bisher ungekanntem Maß ausgeweitet, was zu Umstellungen in den Arbeitsabläufen und zu neuen Arbeitsformen in räumlich getrennten Teams führte (Schmitz 2020). Mit dem Auftreten einer zweiten Krankheitswelle ab Herbst 2020 und darauf folgenden zunehmenden Restriktionen, bis hin zur zweiten Schließung zahlreicher Organisationen der Freizeit-, Veranstaltungs- und Tourismusbranchen sowie von Bildungs- und Sportorganisationen ab Dezember 2020, wuchs das Unbehagen in der Öffentlichkeit mit der erneuten Ungewissheit: Wie lange wird das dauern? Wie wird sich mein Lebensumfeld dadurch ändern? Kann mein bisheriges Arbeitsleben aufrecht erhalten werden?

Die gemeinsame Sinnkonstruktion in den sozialen Systemen scheint nur noch begrenzt zu tragen und die erlebte Ungewissheit führt zu Handlungsunsicherheit und stellt für Einzelne ihre Identitätskonstruktionen und ihr soziales Rollenset in Frage. Dies gilt im Negativen wie im Positiven. Für Viele wurde die räumliche Isolierung und das Zurückgeworfen-Sein auf die Kernfamilie als Verlust an Autonomie und Einbuße an Handlungskompetenz erlebt - im Zweifelsfall auch als Überforderung (wenn z.B. neue Rollenanforderungen an Eltern herangetragen werden, die ihre Kinder nun im Home-Schooling unterrichten sollen). Für Andere bedeuten die Bedingungen des Arbeitens im Home-Office eine (zumindest temporäre) Aufhebung der Trennung von Haushalt und Betrieb, mit neuen Anforderungen, die sowohl Chancen bergen als auch die Gefahr der Entgrenzung von zeitlichen und inhaltlichen Arbeitsanforderungen mit sich bringen können. Für Manche bedingt die Schließung von Organisationen (speziell im Dienstleistungsbereich) eine Trennung von der Teilhabe am Erwerbsleben durch Verschließung der Erwerbsräume. Hier werden Ungewissheitserfahrungen produziert, die mit dem jeweiligen sozialen Feld auch den Sinnraum des sozialen Handelns verändern es werden neue Repräsentationsräume geschaffen.

Mit Repräsentationsräumen werden kulturelle Symbolsysteme bezeichnet, die kollektiv konstruiert und in der Lebenswelt verankert sind (Günzel 2018). Dies lässt sich von Raumrepräsentationen, die an Produktionsverhältnisse gebunden sind, und der Raumpraxis, als subjektiv gelebte Praxis sozialen Handelns in diesem Kontext, unterscheiden. In Bezug auf Organisationen bedeutet das, dass die
Raumpraxis in den tradierten Strukturen, Hierarchien und Orten des Arbeitslebens, in den alltäglichen Bedingungen des Arbeitens stattfindet. Die Raumrepräsentation ist der Sinnverweis der überformenden Organisationen: „Hier in unserer Firma machen wir das so, das ist unsere Mission." Dies sind Verweise auf den Sinngehalt eines institutionellen Settings, der eine Organisation prägt und als Ausdruck der Organisationskultur verstanden werden kann (Elbe 2016). Mit der Raumperspektive wird Organisationskultur als Wissens-, Handlungs- und Symbolzusammenhang nun über strukturelle Gebundenheit hinaus erweitert. ${ }^{3}$ Der Repräsentationsraum der Organisation war auch in der modernen Arbeitswelt an die Raumrepräsentation (häufig an einem bestimmten Ort, z.B. das Büro) gebunden und wurde in der Raumpraxis reproduziert. Rollen waren raumgebundene Repräsentationen des Ichs und die soziale Identität (Elbe 2013; Tajfel und Turner 1986) daran geknüpft. Dies ändert sich nun für Viele: Die Arbeitserbringung ist nicht möglich (z.B. im Gast- oder Veranstaltungsgewerbe) oder findet zuhause statt (z.B. an einem Telearbeitsplatz). Sowohl Raumrepräsentation als auch Raumpraxis ändern sich - plötzlich sind Arbeitsorganisation und Zuhause nur noch temporär zu trennen und die Rollenanforderungen der privaten und beruflichen Sphäre greifen in einander über. Die Rückkehr zum status quo ante scheint zweifelhaft. Das gilt sowohl für den Sinnraum der Organisation - als Teil des privaten Wohnbereichs oder projektförmige Substitution von Linienstrukturen - als auch für die Identitätskonstruktionen der Betroffenen.

Die soziale Identität und das Rollenverhalten in Temporären Organisationen entwickeln sich unter dem Einfluss von Ungewissheit in Korrelation zueinander. Es liegt keine einfache, kausale Beziehung vor, vielmehr wird die Gestaltung der Organisation als Möglichkeitsraum beruflicher Tätigkeit darüber entscheiden, inwieweit sich Mitarbeiter und Mitarbeiterinnen der Organisation gegenüber als beitragsfähig und verpflichtet fühlen. Ungewissheit ist nicht nur Grundlage der Handlungsermöglichung sondern auch der emotionalen Bewertung - es ist die „Geborgenheit des Menschen in seiner Ungeborgenheit" (Wust 1940, S. 301), die ihn auch unter Ungewissheitsbedingungen an die Organisation bindet.

\footnotetext{
3 Als strukturelle Gebundenheit sind hier z. B. örtliche oder soziale Bindungen zu verstehen, die den Umgang miteinander und die Kontextualisierung von Situationen prägen. In der klassischen Organisationskulturforschung wurde dies vielfach als Cultural Complexity (Sackmann 1997) thematisiert, in der regionale oder nationale, branchenspezifische oder professionelle, ethnische oder abteilungsspezifische kulturelle Muster aufeinander treffen. Aus der Raumperspektive kann dies nun als jeweils neu ausgehandelter Sinn-Raum verstanden werden, der einer gemeinsamen Situationsdefinition dient, wobei die strukturellen Einflüsse in den Hintergrund treten. Trotzdem gibt es einen gemeinsamen Sinnhorizont und eine Kooperationsgrundlage, die auf Zusammengehörigkeitsgefühl beruht.
} 


\subsection{Temporäre Identität und kognitive Karten}

Aufgrund der generellen Erosion des traditionellen Normalarbeitsverhältnisses (Elbe 2013) sowie der veränderten Raumkonzeption von Organisationen in der Pandemie verändern sich auch die Identitätsbezüge und die damit verbundenen biographischen Erzählungen. Eindeutig vorgegebene Laufbahnen gibt es immer weniger und Routinearbeiten, die ermüden, zugleich aber beruhigen, verlieren an Bedeutung (Bauman 2008, S. 91). Mit der Zunahme von Wissensarbeit, räumlicher Trennung und Projektstrukturen zeigen sich bereits seit längerem Veränderungen in der Arbeitsorganisation, die mit den aktuellen Ungewissheiten ein ungekanntes Ausmaß erreichen. Wo bisher einmal erworbene Kompetenzen dauerhaft in einer Organisation und im Zweifel auch auf dem Arbeitsmarkt eine konstante Ressource darstellten, auf die Arbeitnehmerinnen und Arbeitnehmer setzen konnten, hat nun die Ungewissheit über zukünftige Arbeitsanforderungen und -bedingungen eine Veränderung der Arbeitsbeziehungen und der organisationalen Sinnkonstruktionen erzeugt, die bei Vielen ein Gefühl von Ohnmacht erzeugt.

Die klassische Unsicherheitsreduktion durch Kontrolle funktioniert nicht mehr, zugleich fällt es vielen Betroffenen schwer, neue Ressourcen in der Offenheit des Ungewissen zu finden. Dies gilt umso mehr, als eben diejenigen, die sich in hohem Maß an die Fluidität der neuen Arbeitswelt angepasst hatten (z.B. als Solo-Selbständige) vielfach in besonderem Maß von den wirtschaftlichen Auswirkungen der Pandemie betroffen waren und nicht über das soziale Netz einer traditionellen Arbeitsbeziehung verfügten. Der Verlust sozialer Identitätsbezüge erweist sich vor dem Hintergrund des Wandels in der Arbeitswelt als eine Herausforderung. Die Entwicklung eines proaktiven, unternehmerischen Selbst wird zur zentralen Anforderung auf dem Arbeitsmarkt (Keupp und Dill 2010, S. 10). Der oder die Einzelnen muss auf kompatible Identitäten in wechselnden Raumkonstellationen der Organisation als Ausweis der Beschäftigungsfähigkeit zurückgreifen können. Die soziale Identität kann sich nicht mehr dauerhaft aus einer Gruppenoder Organisationszugehörigkeit ableiten (Elbe 2013; Tajfel und Turner 1986), vielmehr entsteht die Notwendigkeit sich wandelnde Rollen in unterschiedlichen raumgebundene Repräsentationen einer oder mehrerer Organisationen in einer Bastel- oder Patchwork-Identität (Keupp 1997) zu vereinigen. Da aber, aus psychologische Sicht, Anerkennung und Zugehörigkeit grundlegende Aspekte des Identitätskonstrukts sind (Keupp 1997, S. 34), sind die partiellen Identitätsangebote, die Organisationen mit ihren Repräsentationsräumen - auch im Home-Office oder in Projektstrukturen - bieten können, zeitlich gebunden. Es sind geliehene, temporäre Identitätsschnipsel, die einer umfassenden Klammer bedürfen. Der Company Man lebte in der Werkswoh- nung (Elbe 2013), der Patchwork Man hingegen arbeitet im Home-Office. $^{4}$

Verstehen wir Raum als ,relationale (An)Ordnung sozialer Güter und Lebewesen an einem Ort" (Löw 2019, S. 212), dann wird klar, dass sich Organisation - als Koordinationsform relationaler Ungleichheiten - sowohl hinsichtlich der Kompensation von Ungewissheit als auch der kollektiven Identitätsstiftung durch Virtualisierung und Verprojektlichung temporalisiert und nur mehr partiell koordinative Wirkung (und Sinnstiftung) entfalten kann. Eine entsprechende Klammer für die individuell entstehende Bastelidentität liefert stattdessen die Employography: Die Unsicherheitsfelder der Arbeitswelt können reduziert und die Vielzahl an Identitätsfragmenten integriert werden, wenn die eigene Erwerbsbiographie zur handlungsleitenden Institution und zum zentralen Identitätsbezug wird und damit „,zum dauerhaften Bezugspunkt der sozialen Identität“ (Elbe 2013, S. 18). Employography (als Zusammenziehung des englischen Begriffs für Anstellungsfähigkeit: Employability mit dem eigenen Lebenslauf als Bezugsrahmen der Identiätsarbeit: Biography) kennzeichnet eine Institutionalisierung der Selbstinszenierung und der Autonomisierung des Individuums, wenn organisationale Bindungen nicht mehr dauerhaft tragen. In einer umfassenden Untersuchung hat Elbe (2019) dies anhand der Karrieren ehemaliger Bundeswehroffiziere analysiert. Aufgrund der Befragung von 1028 ehemaligen Offiziere konnte gezeigt werden, dass diese die recht unterschiedlichen Karriereabschnitte im Militär und in der zivilen Arbeitswelt doch als eine geschlossene Karriere erleben und sich an ihrer Employography als Karrieremuster orientieren und nicht an einzelnen organisationalen Anforderungen. Mit dieser Untersuchung wurde eine Studie von Marr (2002) bestätigt, der dies - unter Mitarbeit von Elbe, Morick und weiteren Beteiligten - auch schon für den Zeitraum von 1980 bis 2000 feststellen konnte.

Die organisationale Ankoppelung kann nur noch temporäre Identitätsfragmente anbieten. Diese können mit Hilfe von kognitiven Landkarten wieder integriert werden. Weick und Bougon (1986) konzipieren - im Anschluss an Kurt Lewin - organisationale Wissenslandkarten, wobei individuelle kognitive Landkarten mit sozialen Bezügen kombiniert werden. Kognitive Karten bezeichnen in der Psychologie mentale Repräsentationen räumlicher Relationen, sie die-

\footnotetext{
${ }^{4}$ Eine konkrete, anteilige Zuschreibung eines Ungewissheitsempfindens zu Lasten einer allgemeinen Identitätsdiffusion, generellen gesellschaftlichen Wandels in der Moderne oder anhaltenden Veränderungen in der Arbeitswelt (Erosion des klassischen Normalarbeitsverhältnisse, Digitalisierung und Virtualisierung) sowie spezifische Krisenerscheinungen (wie aktuell die Corona-Pandemie) ist weder notwendig noch sinnvoll und ggf. auch gar nicht möglich. Zentral ist die Diagnose zunehmenden Ungewissheitsempfindens, das mit all diesen Erscheinungen verbunden ist und sowohl Belastungen als auch Entwicklungschancen in sich birgt. Der diagnostische Erkenntnisprozess bedingt Bewusstwerdung, die Lernchancen erzeugt (Elbe 2015).
} 
nen dem Individuum zur Strukturierung von Lebensräumen und bieten damit eine Orientierungsgrundlage zur Verhaltenssteuerung (Downs und Stea 2019). Dies zeigt sich z.B. in der veränderten Bedeutung von Körperlichkeit im Rahmen der zunehmenden Virtualisierung. In digitalen Räumen sind Menschen in ihrer Körperlichkeit reduziert, digitale Besprechungen zeigen nur einen Ausschnitt vor einer bewusst gewählten Umgebung. Besondere körperliche Attribute (denen eine einnehmende oder irritierende Wirkung zugeschrieben werden können) bleiben vielfach ausgeblendet, der Fokus ist auf das Gesicht und den Oberkörper reduziert. Nonverbale Kommunikationsanteile werden auf die Mimik beschränkt, Gestik und weitere Körpersprache sind weitgehend ausgeblendet. Die Inszenierung des eigenen Auftritts wird von der Wirkmöglichkeit reduziert, was dazu führt, dass ,die Technik der Videokonferenz eine gewisse Nivellierung mit sich bringt." (Herzog 2021, S. 5) Die Formen der Selbstinszenierung verändern sich und damit auch die sozialen Bezüge. Für die Einzelnen führt dies zu einer neuen Wahlfreiheit hinsichtlich der Wirkung im digitalen Resonanzraum. „Aus dieser Wahlfreiheit ergibt sich die Herausforderung der ständigen Persönlichkeitsbildung: Wer will ich sein? Und für wen?“ (Tholl 2021, S. 5) Die Selbstinszenierung erzeugt Bezüge in der kognitiven Selbstverortung, die sozial-digitalen Rollen werden anhand entsprechender kognitiver Landkarten präsentiert.

Wie kann dies nun im Rahmen eines Managements von Ungewissheit in organisationalen Kontexten berücksichtigt werden? Im Rahmen des Wissensmanagements sowie der Lerntheorie finden Strukturierungs- und Visualisierungstechniken wie Cognitive map, Mnemo-Technik und Mind Mapping seit langem Anwendungsfelder, um Raumeindrücke mental zu verorten, Wissensstrukturen zu visualisieren und Vorstellungsräume abzubilden (Seifert 2005). Weick und Bougon (1986) wollen durch ihre organisationalen kognitiven Karten nun die Zugänglichkeit und Bedeutsamkeit organisationaler Wissensbestandteile strukturieren. Dies ist grundsätzlich nicht neu, so sind Organigramme nichts anderes als Repräsentationen sozialer Beziehung nach bestimmten Kriterien (z.B. Kommunikationsregeln wie Anordnungsbefugnis und Informationspflicht), die sich durch Ausweitung der Kriterien und assoziative Verknüpfungen zugleich komplexer und flexibler gestalten lassen. Die kognitiven Karten, die wir uns von Organisationen machen, legen die sozialen Beziehungen und ihr evolutionäres Potenziale besser offen, als es traditionelle Organigramme können. Dies wird unter den neuen Bedingungen des Arbeitens aber nur gelingen, wenn die Organisationen den Mitarbeiterinnen und Mitarbeitern dauerhafte Identitätsangebote liefern (Elbe 2013), die sich in deren Employography integrieren lässt - auch in Projektstrukturen oder unter virtuellen Bedingungen des Zusammenarbeitens. Die Bereitstellung der hierfür not- wendigen Raumkonstellationen erfolgt im Rahmen der Temporären Organisation.

Die Temporäre Organisation ist als Grundperspektive auf das, was Organisation letztlich ausmacht, zu verstehen: die Absicherung von sicherungsbedürftigen Kooperationsbedingungen (Elbe und Peters 2016). Diese bedarf einer immerwährenden Neujustierung in der Abstimmung der drei Faktoren Prozess, Struktur und Institution. Eine relationale Verortung in diesem Konstrukt gelingt, wenn die einzelnen Komponenten als voneinander abhängig begriffen und in einer kognitiven Karte miteinander in Bezug gesetzt werden. Hierdurch gelingt es sowohl den einzelne Mitarbeitern und Mitarbeiterinnen als auch der Organisation, Handlungsprobleme als bewältigbar und Ungewissheit als Entwicklungschance zu erleben (Elbe 2013). Handlungsoptionen und Entscheidungen beschränken sich unter diesen relationalen Bedingungen nicht auf feste Verfahrensabläufe und Regeln, sondern bieten die Möglichkeiten bei Bedarf unterschiedliche Bezugsfelder zu aktivieren und im Zweifelsfall Prozesse, Strukturelemente oder institutionelle Gegebenheiten jeweils zu substituieren. Durch kognitive Landkarten lassen sich Ungewissheitspotenziale in der organisationalen Identität verankern, statt z. B. durch weitere Katastrophenpläne zukünftige Unsicherheiten beherrschen zu wollen, ${ }^{5}$ womit im Zweifelsfall Innovationen behindert würden, wenn sie sich als ungeeignet herausstellen, eine aktuelle Krisensituation zu managen (Elbe 2013).

\section{Neue Rollen in der Temporären Organisation}

\subsection{Wissensarbeit in der Temporären Organisation}

Im Folgenden konzentrieren wir uns auf die Entwicklungstendenzen der Temporären Organisation (Elbe und Peters 2016). Mit der Konzentration auf diese Perspektive greifen wir die aktuellen Entwicklungen auf, die mit dem Begriff der Digitalen Transformationen (Boes et al. 2018; Reinhardt 2020) verbunden sind und schon vor dem Auftreten der Corona-Pandemie einen grundlegenden Umbruch in Organisationen und Arbeitsformen markiert haben. Digitalisierung und Wissensarbeit nehmen seit Jahren zu und damit wird der Fokus auf neue Arbeitsformen (z.B. hinsichtlich der organisationalen Einbindung) und Arbeitsmittel (z.B. Telearbeitsplätze) gelenkt. Die organisationalen Raumkonzepte verändern sich und es bilden sich neue Tätigkeiten und

\footnotetext{
5 Dies spricht nicht grundsätzlich gegen Katastrophenpläne oder Planung generell, nur sollte auch hierbei von einem abnehmenden Grenzertrag ausgegangen werden - bevor die Grenzrate Null erreicht sollte auf ein bewusstes Ungewissheitsmanagement (Böhle und Busch 2012) umgestellt worden sein.
} 
Aufgabenzuschnitte heraus, wodurch bisher vertikal organisierte Arbeitsteilung neu verteilt und koordiniert wird. Der Wandel von Arbeit realisiert sich nicht mehr nur in der Gestaltung technisch-sozialer Räume, sondern stößt mit Informatisierungsprozessen und Projekten selbst Veränderungen an. Damit stehen auch die Rollen klassischer Prozessverantwortlichkeit zur Disposition und werden gleichsam verprojektiert (Peters und Dengler 2010; Peters 2012, S. 53 f). Die Neuausrichtung von Räumen und Rollen ist zunehmend gebunden an Informationstechnologie und Wissensarbeit, z. B. durch die Kartographisierung von Wissen, wobei der Umgang mit Ungewissheit ein Grundthema darstellt (Schnauffer et al. 2014).

Alle Organisationsprozesse stehen prinzipiell zur Disposition, betroffen sind allerdings vor allem Beschäftigtengruppen in Bereichen, in denen es um das Erbringen immaterieller Leistungen (z. B. Design, Finanzen, Gesundheitswesen) geht und die durch Informationskomplexität mit hohen Ungewissheitspotenzialen geprägt sind (vgl. z. B. Böhle und Busch 2012; Jeschke et al. 2011). Neue Rollen entstehen insbesondere in fluiden Prozessstrukturen, die zur Etablierung der Digitalisierung, als zentralem Transformationstreiber, beitragen und die Kollektivierung der Wissensarbeit in Organisationen vorantreiben. Hierfür bedarf es der relationalen Vernetzung und kognitiven Abbildung des Bezugsfeldes, das durch agilen Wissensaustausch untereinander in seinen fluiden Eigenschaften konstituiert wird. Ziel sollte es sein, eine neue systemische Integration der betroffenen Beschäftigtengruppen zu erreichen und diese - unabhängig von ihrer räumlichen Verortung - in das gemeinsame kognitive Kartengeflecht einzubinden. Verbunden hiermit sind neue Gruppenrollen, bei denen nicht der individuelle Kompetenznachweis im Vordergrund steht, sondern die Anforderungen aus der temporären Prozessbearbeitung (Elbe 2016). Rollen können dabei als Teile der sozialen Identitätskonstruktion begriffen werden, die sich an bestimmte soziale Positionen binden und die aufgrund ihres Erwartungscharakters mit den neuen Arbeitsformen hinsichtlich des Anleitungsbedarfs oder der Weisungsgebundenheit eine neue Ausrichtung erfahren. Der Fokus innerhalb der digitalen Transformation, die durch das Pandemiegeschehen 2020/2021 noch verstärkt wird, richtet sich insbesondere auf die Gestaltung von Prozessstrukturen. Speziell die Ablauforganisation und die Prozesse der Zusammenarbeit werden durch die Veränderung der Arbeitsbedingungen neu verhandelt. Dies bedingt eine Anpassung der Rollen die mit (nunmehr) temporären Prozessen verbunden sind. Damit sind auch neue institutionelle Muster der Einbindung aller Beteiligten, insbesondere aber der Organisationsmitglieder, zu erproben (Salazar und Peters 2011).

Temporäre systemische Eingriffe mit neuen Öffnungen der Zusammenarbeit in den privaten Bereich (z. B. im Rahmen von Home-Office), aber auch in Projektstrukturen er- zeugen Prozessformen, worin Mitglieder temporär selektive Veränderungen ihres Expertenmodus erfahren und damit auch einen Wandel der Rollenerwartungen. Aufgrund der digitaler Trends - und der gesellschaftlichen Ungewissheit im Zuge der Corona-Pandemie - bleiben die Prozesse und Strukturen, die aktuell Kooperation ermöglichen, temporär und projektorientiert. Eine Verstetigung scheint ungewiss, da weiterhin mit erheblichen Veränderungen gerechnet werden muss. In diesen temporären Strukturen erlebt die Arbeitswelt ihre Umwälzungen und viele Gewissheiten lösen sich auf. Im Kontext der Informatisierung ist dies zunächst ein sozialer Vorgang mit dem Ziel, entsprechende Tätigkeiten und ihre Ergebnisse anderen Experten zugänglich zu machen (Boes et al. 2018), bevor Entscheidungen gefällt werden müssen. So wird der Fokus nunmehr auf Informatisierungsräume als entscheidendem Faktor gelenkt, in denen der Austausch von Wissen zentral ist. Die Temporalität der Organisation (Elbe und Peters 2016) ist in diesem Veränderungsprozess auf Struktur-, Prozess- und Institutionenebene gleichzeitig angesprochen - wir haben es hier also nicht mit partiellen Substitutionsprozessen zwischen zwei organisationalen Bereichen zu tun, wie sie bisher den Wandel in Organisationen kennzeichnen, sondern mit einem umfassenden Innovationsprozess, der alle organisationalen Sektoren (den primären staatlichen, den sekundär privatwirtschaftlichen und auch den tertiär gemeinnützigen) betrifft.

\subsection{Ver-Projektierung und Wandel der Rollen}

Während Organisation Regelhaftigkeit - und damit Sicherheit vor unverbindlicher Kurzfristigkeit und marktlichen Aushandlungsprozessen - bieten soll, schaffen Projekte in Organisationen eben das: eine ,,verbindliche Kurzfristigkeit" eingebettet in eine gesicherte sozio-ökonomische Beziehung. Mit der Ver-Projektierung in der Wissensarbeit hält die Ungewissheit erneut Einzug in die Organisation, wirkt nun aber flexibilisierend und innovationsfördernd. Zum einen werden Arbeitsprozesse dadurch weniger berechenbar (sie verlieren einen Teil ihrer Sicherungsfunktion für alltägliche Handlungsabläufe), zum anderen aber schafft eben dies Flexibilität hinsichtlich der Ausgestaltung neuer Rollenarrangements (z.B. zwischen Arbeitsleistung im Home-Office und sich verändernden familiären Anforderungen) und Identitätskonstruktionen.

Dies betrifft auch die Frage der Führung: Führungstätigkeit in klassischen Organisationsformen stand im Spannungsfeld zwischen institutionalisierter Herrschaft (als Hierarchie) und informeller Führung in Gruppenprozessen (Elbe 2016). Im virtuellen Raum sinkt einerseits die erlebte Symbolik hierarchischer Unterschiede (z.B. zum "Chef“ gerufen zu werden, sich körperlich dorthin zu begeben, dem symbolisierten Statusgefälle in der Größe und Ausstattung des Büros - insgesamt also den kulturbedingten, geron- 
nenen Symbolen sozialer Ungleichheit und Unterordnung ausgesetzt zu werden und damit einer spezifischen Situationsdefinition) hin zu einer egalitäreren Interaktionsordnung (jetzt bleibt man im eigenen Arbeitszimmer, ist über eine virtuelle Plattform verbunden und der symbolischen Dominanz entzogen - ggf. auch einer körperlichen Dominanz). Andererseits steigt die Bedeutung informeller Führung, da Führung nun in viel höherem $\mathrm{Maß}$ vier Funktionen wahrnehmen muss: Moderieren, Coachen, Trainieren und Mentor sein (Oestereich und Schröder 2020). Diese neuen Führungsaufgaben erfordern eine Begegnung auf Augenhöhe, die nicht durch Hierarchie begründet werden kann, sondern vielmehr eine entsprechende Kompetenzzuschreibung als Grundlage des Führungsverhaltens notwendig macht.

Im virtuellen Raum werden Führungsfunktionen vielfach mit Art Influencer-Rollen verbunden, die aber - statt Livestyle - Sinnangebote im Kooperationskontext vermitteln. Durch diesen informellen Charakter führt der Wandel von Führungsaufgaben zu einer Rollendiversität, die kaum mehr auf Hierarchie zurückgreifen kann. Im Rahmen von wechselnden Projektstrukturen ist immer wieder neu zu klären, welche Rollen mit Führungsaufgaben verbunden werden und welche Rollen im Expertenmodus eingenommen werden. Die Komplexität und Ungewissheit in Digitalisierungsprozessen führt dazu, dass Gruppenaufbauund Gruppenaufgabenrollen zu unterschiedlichen Zeiten im Verlauf von Projekten von verschiedenen Personen in unterschiedlichem Maß wahrgenommen werden (Elbe 2016, S. 96f). Hierbei ist von (virtuellen) Gruppen als offenen Sozialsystemen auszugehen, die auf diesen Austausch im Sinne einer Selbststeuerung der Gruppe angewiesen sind und zwar nicht nur aus Effizienzgründen, sondern auch um die Gruppe zu stabilisieren. ${ }^{6}$ Dies bedeutet nicht, dass es keinen personellen Austausch von Gruppenmitgliedern mit anderen Projektgruppen in der Organisation geben könne. Ein Austausch von Rolleninhabern innerhalb der Organisation sollte sogar erfolgen, wenn einzelne Personen zu sehr auf bestimmte Rollen festgelegt sind und damit das Entwicklungspotenzial der Gruppe gehemmt werden könnte.

In unterschiedlichen Rollenzuschnitten werden Befindlichkeiten und Selbstansprüche von Mitarbeiterinnen und Mitarbeitern ausgelotet. Wechselnde Gruppen geben dem Sinn der Tätigkeit in der Organisation neue Impulse, wodurch generelle Innovationsfähigkeit ermöglicht wird

\footnotetext{
${ }^{6}$ Auch virtuelle Gruppen sind von gruppendynamischen Prozessen geprägt, die damit - hier ist Krainz (2020, S. 489) zuzustimmen - ein „Jenseits des Marktprinzips“ ermöglichen und damit zum einen zutiefst (mikro-) politisch sind, zum anderen aber - und hier ist Krainz (2020, S. 496) zu widersprechen - natürlich ihre Wurzeln in der Sozialpsychologie Lewin'scher Prägung haben. Eben hierdurch wird der soziale Wandel zur Angelegenheit aller Betroffenen, die damit auch dauerhaft zu Beteiligten werden (Elbe und Erhardt 2020).
}

(Jeschke et al. 2011; Oestereich und Schröder 2020) und Mitarbeitende zu Akteuren werden, die in ihren Rollenarrangements neue Kopplungen von Prozessen und organisationalen Zielen bearbeiten (Elbe und Peters 2016; Peters und Dengler 2010). Innerhalb dieser flexiblen Entwicklungen hat die Zerlegung von Aufgaben in verschiedenen Prozessphasen erhebliche Bedeutung, weil die Verantwortungsbereiche aus der Geschäftsebene in die Projektbereiche abwandern. Rollenträger übernehmen im Rahmen der Selbstorganisation notwendige wiederkehrende Prozesse und inszenieren diesen Aufgabenteil nun öffentlich. Aufgrund des zunehmenden Wegfalls örtlicher Bindung (insbesondere im Rahmen der Virtualisierung in Folge der Corona-Pandemie) verändert sich die Form sozialer Kontrolle in Organisationen. Formale Kontrolle wird vielfach informalisiert und temporalisiert, d.h. in wechselnde Rollen verteilt. Damit werden Verhandlungspotenziale geschaffen, die im Rahmen der Abstimmung temporärer Aufgabenbereiche mit anderen Organisationsteilen eingesetzt werden können. Zugleich bilden sich projektspezifisch neue Gruppenrollen heraus, von denen hier drei typische genauer dargestellt werden sollen (Oestereich und Schröder 2020, S. 117; Salazar und Peters 2011):

a) Gastgeberrollen, die regelmäßig zu Entscheidungstreffen, bzw. deren Vorbereitung einladen,

b) Dokumentarrollen, die gemeinsam getroffene Entscheidungen festhalten und aktualisieren, damit alle informiert bleiben und Vereinbarungen eingehalten werden,

c) Lernbegleiterrollen, die dazu anregen über die gemeinsame Arbeit und Selbstorganisation zu reflektieren und daraus zu lernen sowie regelmäßig Retrospektiven veranstalten, um Verbesserungen zu vereinbaren und Lernprozesse zu initiieren.

Es ist nun keineswegs so, dass diese Rollen formal abgegrenzt wären, Überschneidungen und Doppelungen sind gegeben. Es ist eher eine heuristische Trennung neuer Erwartungssets, die aber die klassischen Gruppenaufbauund Gruppenaufgabenrollen differenzieren, bzw. ablösen. Neue Tätigkeiten und Aufgabenzuschnitte unterliegen dabei selbst der Temporalität. Die Gastgeberrolle kann z.B. den verschiedenen Prozessphasen für die Bearbeitung vorgeschaltet werden und gleichzeitig aus dem Verantwortungsbereich der Geschäftsebene in die Projektbereiche abwandern. Dabei kommt der Entscheidungsvorbereitung eine Bedeutung $\mathrm{zu}$, die bisher insbesondere die Gestaltungsebene prägte, nun aber zur partiellen Sinnstiftung der Projekterfolge beiträgt. Darüber hinaus wird der Zugang zu organisationalen Wissensbeständen neu geregelt. Entscheidungsvoraussetzungen werden nun differenzierter bewertet und festigen die Raumrepräsentationen in verschiedenen Projektphasen der temporärer Projektbearbeitungen. 
Typische Gastgeberrollen finden sich z. B. in organisationsinternen Kampagnen, bei denen Themen gewählt werden, die von den Beteiligten eine hohe Relevanz zugesprochen bekommen haben. Parallel können somit verschiedene Entwicklungsoptionen aufgegriffen und vorangetrieben werden. Kampagnen werden von einem temporären Teamleiter verantwortet (Daum et al. 2020). Ähnliche differenzierte neue Raumpräsentationen zeigen Entwicklungen innerhalb der Dokumentarrolle, sie deckt insbesondere Rollenmodelle und Methoden ab, in denen lessons learned-Erfahrungen festgehalten und in andere Bereichen zur Informatisierung oder für veränderte Zusammenarbeit genutzt werden. Bei der Lernbegleiterrolle geht es um den Kompetenz- und Wissenszuwachs der Mitglieder und ihre Begleitung, ${ }^{7}$ z. B. hinsichtlich „kleiner" Lernschleifen und Meilensteine, die dabei helfen über die eigene Arbeit und Selbstorganisation zu reflektieren und daraus zu lernen. Hochqualifizierte Mitarbeiter und Mitarbeiterinnen erwarten für eine Lernbegleitung vielfach das Coaching und entscheiden dann, ob Weiterbildung im jeweiligen Fachgebiet favorisiert oder der Schwerpunkt auf das Lernen im Prozess der Arbeit gelegt wird (Peters et al. 2016). Mit dem Wandel der Rollen verändert sich so auch die Raumpraxis und passt sich dem Wandel der Organisation als Repräsentationsraum an (Salazar und Peters 2011). Lernförderliche Arbeitsgestaltung wandelt sich dabei von einem Innovationstreiber in herkömmlichen Organisationsstrukturen zu einem zentralen Merkmal neuer, virtualisierter Projekträume. Die Informatisierung bedingt ein eigenständiges Weiterlernen im Prozess der Arbeit und verbunden damit eine Weiterentwicklung der Organisation als Sinnraum. Hierbei haben sich im Zuge des agilen Projektmanagements (Elbe und Peters 2016, S. 102) spezifische Rollenbezeichnungen (z.B. Scrummaster) herausgebildet, die den Wechsel von Führungspositionen und anderen Rollen kennzeichnen. Neben klassischen Formen der Unterstützung zur Weiterentwicklung von Organisationsmitgliedern, die in temporären Projektstrukturen arbeiten, wie dem Mentoring, haben sich neuere Ansätze wie das Nudging (Schoper et al. 2020) gesellt. Dies trägt der Vorstellung Rechnung, dass die Integration spezifischer Mitarbeiterinnen und Mitarbeiter selbstmotiviert erfolgen muss, wohl aber eines Anstoßes im Sinne eines leichten Schupsens bedürfe, um sich aktiv an Veränderungsprozessen zu beteiligen und spezifische Rollen zu übernehmen, die alle insgesamt transparenter sind.

\footnotetext{
${ }^{7}$ Dazu gehören auch sogenannte Wissenspromotoren, die für Wissens(ver)teilungsprozesse eine wichtige Rolle einnehmen (Peters und Dengler 2010).
}

\subsection{Temporäre Rollen und temporäre Räume}

Rollen erfahren eine Temporalisierung, sie werden weniger regelgebunden und explorativer (Elbe 2016). Damit verlieren sie aber an Verbindlichkeit für die Organisation, sie gelten nur im Projekt und wenn sie Gültigkeit darüber hinaus beanspruchen, dann stellt das die Sinnkonstruktion der Organisation, ihren Repräsentationsraum in Frage. Die Temporalisierung der Rollen ist Ergebnis einer zunehmenden Temporalisierung der Organisation an sich, die durch die Verzeitlichung der Struktur in Projekten auch die Sinnbezüge und sozialen Identitätsangebote in jeweils eigene Projekträume fasst. Ungewissheitsressourcen entstehen dabei durch die Zunahme an Vernetzungen in der Organisation. Durch die Zunahme vernetzt-verteilter Arbeit (Daum et al. 2020) wächst der Anteil immaterieller Arbeitsgegenstände, die in Projektorganisation bearbeitet werden. Dort, wo der Nutzen und Umgang mit Wissen in temporären Arbeitsprozessen geplant, entwickelt, ausgetauscht, gestaltet und bewertet wird, ist Transparenz geboten, um Ungewissheit aufzufangen. Alle Projektbeteiligte sind zu involvieren, um entsprechende Informatisierungsräume aufzubauen, die zum einen Schutzräume des Austauschs sind, zum anderen aber Veränderungen in kleinen Schritten ermöglichen. Diese können bei ausbleibendem Nutzen sofort wieder zurückgenommen, bzw. verändert werden. Der Vorteil einer solch evolutionär ausgerichteten Gestaltungsinitiativen (Peters 2012, S. 137) ist darin zu sehen, die innerhalb von Wissensarbeit auftretenden Fragen parallel zu den zu bearbeitenden Kernprozesse bearbeiten zu können. Im Zuge der AGIL-Bewegung (Elbe und Peters 2016; Wagner und Rieteke 2014) sind es eher Werte, Haltungen und verbindliche Prinzipien, an denen sich die Teammitglieder orientieren wollen. ${ }^{8}$ Oesterreich und Schröder (2020) plädieren dafür, Vorbereitung, Gestaltung und möglichen neuen Nutzen in Foren zur Team-Selbstorganisation zu informatisieren, um der Raumpraxis eine Grundlage zu geben und weitere Implikationen (z.B. Rechtefrage in Nutzung und Gebrauch) geklärt zu haben.

Nicht nur durch die Zunahme der Projektstrukturen, auch durch die örtliche Distanzierung und die Virtualisierung unter Corona-Bedingungen verschiebt sich der Repräsentationsraum der Organisation. Je gewohnter diese Veränderung wird, desto mehr wandeln sich auch die Rollenerwartungen sowohl innerhalb der Organisation als auch in Bezug auf andere Marktteilnehmer (Nicht-Organisationsmitglieder) sowie das private Umfeld. Private und öffentliche Räume werden durch den organisationalen Raum infiltriert und das hat Auswirkungen auf die organisationsbezogenen Anteile der individuellen Identitätskonstruktio-

\footnotetext{
${ }^{8}$ Dies gilt für Organisationen aller Sektoren; für die Verwaltung z. B Wirth (2020).
} 
nen und Rollenerwartungen. So sind in virtuellen Räumen und Projektstrukturen Fragen der tarifrechtlichen Zuständigkeitsordnungen noch vielfach unklar. Hochqualifizierte Mitarbeiterinnen und Mitarbeiter bleiben formal in der Linienorganisation angebunden, sind aber de facto in vernetztverteilten Projekten in der gesamten Organisation und darüber hinaus (Daum et al. 2020) eingebunden. Diese Perspektive wird für die Tätigkeit im Home-Office erst jetzt virulent und führt zu Fragen wie z. B. danach, wie Personalratsarbeit im Home-Office organisiert werden kann (Albert 2020). Das sind Aspekte, die in der Literatur zu temporären Projektorganisationen bisher kaum diskutiert werden, es wird über Rollen im Sinne von Arbeitsgestaltung gesprochen, nicht aber über die Flexibilisierung von Zuständigkeiten und Verantwortungsbereichen, Rechte und Verpflichtungen in den neuen Räumen der Organisation, die durch die Digitalisierung ermöglicht und durch die Corona-Pandemie beschleunigt werden.

Dass diese Probleme bisher so wenig in der Literatur beachtet wurden, liegt wohl daran, das Unternehmen auch mit hoher und komplexer Projektstruktur an einer langfristigen Bindung ihrer hochqualifizierten Mitarbeiter und Mitarbeiterinnen festhalten. Es kommt hinzu, dass diese Akteursgruppen einerseits die Freiheit der Selbst-Organisation schätzen (z. B. auch hinsichtlich der Führungs- und Lernangebote), andererseits aber die sichernde formale Einbindung in die Linienstruktur als Backup behalten wollen. Dies führt zumindest in der Linienorganisation (und vielfach auch darüber hinaus) zu einer Diffusion der Erwartungen, die an Rolleninhaber in virtuellen und projektgebundenen Strukturen zu stellen sind. Hiermit verbunden sind Problemen auf der Prozessgestaltungsebene, die in der Organisation zunehmend Aufmerksamkeit erfordert, da auch hier Unübersichtlichkeit und Ungewissheit hinsichtlich Entscheidungsbedingungen und Gestaltungsmodi wirksam sind. Raum und Zeit neu auszuloten, sind entscheidende Anforderungen an eine, dem angepasste Organisationsentwicklung (Elbe und Erhardt 2020). So verfolgt die Prozessbearbeitung in Organisationen nicht mehr nur die fachliche und technologische Perspektive, sondern versucht das sozio-kulturelle Klima in einzelnen Organisationseinheiten aufzugreifen (und ggf. zu Ver-Projektieren), um durch die Kleinteilung von Raum und Zeit Ungewissheit in Entscheidungsprozessen zu kanalisieren und nutzbar zu machen. Das erfolgt auch über die Gestaltung neuer Rollen. Ziel dieser Entwicklungen ist, Hierarchie möglichst abwesend erscheinen zu lassen sowie agile, selbstorganisierte Teams stabil in Organisationen einzubinden und damit die Temporalität der Organisation zu institutionalisieren.

Die Anforderung, Ungewissheit und Transparenz aufzugreifen, stellt nicht nur für die Gestaltung der Zusammenarbeit, sondern auch für die Personalarbeit und insbesondere die Personalentwicklung, neue Herausforderun- gen dar. Mögliche Spannungen zwischen klassischen Mitgliedschaftsrollen und neue Rollen in temporären Projekten stellen die überkommenen Beziehungen und Lernanforderungen grundsätzlich in Frage (Wagner und Rieteke 2014). Wie Hofmann et al. (2020) in einer Studie mit 500 Unternehmensvertreten herausarbeiten, ist die Corona-Krise ein Katalysator für agil entwickelte, digitale Leistungserstellungsprozesse, wobei die Zukunft in einer hybriden und flexiblen Arbeitsorganisation gesehen wird. Es ist festzustellen, dass örtliche Flexibilisierung auch zeitliche Flexibilisierung nach sich zieht. Arbeitszeit-Souveränität für die Mitarbeiterinnen und Mitarbeiter (Peters et al. 2016, 2021) ist ein wichtiger Teil dieses neuen Rollen- und Identitätsarrangements, wie die Autoren in einer aktuellen Studie erneut feststellen:

Im Auftrag der Gesellschaft für Projektmanagement (GPM) wurde hierzu ein Kooperationsprojekt an der TU Berlin und dem Nexus-Institut zu neuen Formen von Arbeitszeitselbstbestimmung in digitalisierten Projektwelten durchgeführt. Die Studie zeigt, dass die Projektakteure insbesondere in (Forschungs-) Projekten in Firmen arbeiten, in denen die Berücksichtigung von Wissen aus verschiedenen Disziplinen zentral ist. Wurde in einer ersten Untersuchung von 2016 nach offenen neuen Rollen gefragt, gab es kaum nennenswerte Befunde. 2021 war das Bild differenzierter und es sind nun neue Rollen wie Scrummaster, Product Owner, etc. die die Projektarbeit prägen. Ortsund zeitunabhängiges Arbeiten ist ein Wesensmerkmal von Wissensarbeitern, wobei bereits 2016 über die Hälfte der Befragten in Projekten angegeben hatten, dass dieses Merkmal für sie weitgehend zutreffen würde (ausführlich vgl. Peters et al. 2021).

Zum Repräsentationsraum gehören nun Repräsentationen hinsichtlich selbstbestimmter Arbeitszeiten, örtlicher Mobilität und der Wahrnehmung vielfältiger Rollen. Wenn diese drei Aspekte sinnhaft aufeinander abgestimmt sind, dann kann wohl von einer ,starken“ Organisationskultur gesprochen werden und in dieser sehen Hofmann et al. (2020) den wichtigsten Robustheitsfaktor für Organisationen in der Corona-Pandemie.

\section{Identitätswandel in ungewissen Räumen}

Der aktuelle Identitäts- und Rollenwandel betrifft auch und insbesondere unsere Arbeitsbeziehungen sowie die Organisationen, die den Raum der Arbeitserbringung in der Moderne bilden. Durch die Virtualisierung und Digitalisierung wurde die Temporalität dieser Konstruktion immer deutlicher und Organisationen wurden zu temporären Repräsentationsräumen, die Identitätsarbeit im beruflichen Kontext ermöglichen. Nicht erst die Corona-Pandemie 2020/2021 führte zur Herausbildung neuer Rollen in Temporären Or- 
ganisationen, unter besonderer Berücksichtigung von Projektstrukturen. Durch die räumliche Neupositionierung der Temporären Organisation und den damit verbundenen Wandel der Mitarbeiterrollen in diesen Strukturen und Prozessen kommt der Employography als Ansatz der Identitätsarbeit auf Basis der eigenen Erwerbsbiographie eine immer größere Bedeutung zu. Auch der oder die Einzelne bestimmt aus professioneller Sicht ihre soziale Position nicht mehr anhand festgefügter Rollen in stabilen Gruppen, sondern aufgrund der eigenen Erfahrungen in unterschiedlichen Kontexten. Diese Identitätskonstruktion ist in hohem Maß mit beruflichem Erfolg verbunden und korrespondiert mit den neuen Rollenkonzepten der Temporären Organisation, mit ihren veränderten gruppendynamischen Bedingungen.

In komplexen, in hohem Maß von Ungewissheit geprägten Entscheidungssituationen erweisen sich Menschen mit hohem Selbstvertrauen und der Neigung zu Extraversion sowie zur gedanklichen Exploration in Experimenten als besonders erfolgreich (Hommel und Nattkemper 2011, S. 109). Intelligenz, Motivation, Alter oder Ausbildung hingegen zeigen kaum einen Zusammenhang mit dem Erfolg. Ungewissheitsorientierte Menschen bedienen sich kognitiver Landkarten, um ihre Position in der Temporären Organisation zu bestimmen und ihr Rollenverhalten dementsprechend anzupassen. Speziell in wissensintensiven Berufen rücken damit das Erfahrungswissen und das Lernen in der Arbeit (Bolte und Neumer 2020) in den Vordergrund der betrieblichen Personalentwicklung. Die lernförderliche Arbeitsgestaltung wird eine wichtige Herausforderung für das projektorientierte Management der Ungewissheit, das selbst erst lernen muss, die Arbeitsbedingungen in virtuellen Räumen zu gestalten. Diese Perspektive hatten bereits March und Olsen (1975) angemahnt. Speziell Führungskräfte sehen sich mit neuen Anforderungen konfrontiert (Hofmann et al. 2020): Es wird Lernbedarf für Führungskräfte hinsichtlich Führung auf Distanz - einschließlich der Möglichkeit zu Rollenwechseln - deutlich, damit Führungsleistungen unter Ungewissheit und in Krisen weiter sinnstiftend sein können.

\section{Literatur}

Albert, L. (2020). Personalratsarbeit im Home-Office. Der Personalrat: Personal-Recht im öffentlichen Dienst., 7/8(37), 19-21.

Bauman, Z. (2008). Flüchtige Zeiten. Leben in der Ungewissheit. Hamburg: Hamburger Edition.

Beck, U. (1986). Risikogesellschaft. Auf dem Weg in eine andere Moderne. Frankfurt a. M.: Suhrkamp.

Boes, A., Kämpf, T., Langes, B., \& Lühr, T.T. (2018). „lean“ und „agil“ im Büro. Düsseldorf: Hans-Böckler Stiftung.

Böhle, F., \& Busch, S. (Hrsg.). (2012). Management von Ungewissheit. Neue Ansätze jenseits von Kontrolle und Ohnmacht. Bielefeld: transcript.

Bolte, A., \& Neumer, J. (Hrsg.). (2020). Lernen in der Arbeit. Erfahrungswissen und lenförderliche Arbeitsgestaltung bei wissensintensiven Berufen. München: Hampp.
Bonß, W. (1995). Vom Risiko. Unsicherheit und Ungewissheit in der Moderne. Hamburg: Hamburger Edition.

Clausen, L. (2007). Kommunikationsnetzwerke der Krisenbewältigung - Deutsche Sicht der Internationalen Dekade der Katastrophenprävention. In H.-V. Hoffmann (Hrsg.), Netzwerk Kommunikation in Zeiten der Krise (S. 91-111). Baden-Baden: Nomos.

Clausen, L., \& Dombrovsky, W. (1983). Einführung in die Soziologie der Katastrophen. Bonn: SDV.

Dalbert, C. (2002). Die Ungewissheitstoleranzskala (UGTS). In E. Brähler, J. Schumacher \& B. Strauß (Hrsg.), Diagnostische Verfahren in der Psychotherapie (S. 374-377). Göttingen: Hogrefe.

Daum, M., Wedel, M., Zinke-Wehlmann, C., \& Ulbrich, H. (Hrsg.). (2020). Gestaltung vernetzt-flexibler Arbeit. Beiträge aus Theorie und Praxis für die digitale Arbeitswelt. Berlin: Springer.

Downs, R., \& Stea, D. (2019). Kognitive Karten und Verhalten im Raum. In S. Günzel (Hrsg.), Texte zur Theorie des Raums (S. 397-404). Stuttgart: Reclam.

Elbe, M. (2013). Employography: Flüchtige Identitäten in Zeiten der Ungewissheit. Journal für Psychologie, 3(21), 1-24.

Elbe, M. (2015). Organisationsdiagnose: Methoden - Fallstudien $\cdot$ Reflexionen. Baltmannsweiler: Schneider Verlag Hohengehren.

Elbe, M. (2016). Sozialpsychologie der Organisation: Verhalten und Intervention in sozialen Systemen. Berlin: Springer Gabler.

Elbe, M. (Hrsg.). (2019). Duale Karriere als Institution. Perspektiven ziviler Karrieren ehemaliger Offiziere. Berlin: Berliner Wissenschaftsverlag.

Elbe, M. (2020). Die Einsatzorganisation als „Lernende Organisation“. In E. Kern, G. Richter, D. Müller \& H. Voß (Hrsg.), Einsatzorganisationen. Erfolgreiches Handeln in Hochrisikosituationen (S. 139-165). Wiesbaden: Springer Gabler.

Elbe, M., \& Erhardt, U. (2020). Konstruktive Organisationsentwicklung: Menschen verstehen · Organisationen gestalten $\cdot$ Lernkulturen entwickeln. Baltmannsweiler: Schneider Verlag Hohengehren.

Elbe, M., \& Peters, S. (2016). Die temporäre Organisation: Grundlagen der Kooperation, Gestaltung und Beratung. Berlin: Springer Gabler.

Gigerenzer, G. (2007). Bauchentscheidungen. Die Intelligenz des Unbewussten und die Macht der Intuition. München: Goldmann.

Günzel, S. (2018). Raum. Eine kulturwissenschaftliche Einführung. Bielefeld: transcript.

Helsper, W., Hörster, R., \& Kade, J. (Hrsg.). (2003). Ungewissheit. Pädagogische Felder im Modernisierungsprozess. Weilerswist: Velbrück.

Herzog, L. (2021): Von den Schultern aufwärts. Der Tagesspiegel am Sonntag. Nr. 24.484 vom 21.03.2021, 5.

Hofmann, J., Piele, A., \& Piele, P. (2020). Arbeiten in der CoronaPandemie - Aufdem Weg zum New Normal. Stuttgart: Frauenhofer IAO.

Hommel, B., \& Nattkemper, D. (2011). Handlungspsychologie. Planung und Kontrolle intentionalen Handelns. Berlin: Springer.

Jeschke, S., Isenhardt, I., Hees, F., \& Trantow, S. (Hrsg.). (2011). Enabling Innovation. Innovationsfähigkeit - deutsche und internationale Perspektiven. Berlin: Springer.

Keupp, H. (1997). Diskursarena Identität: Lernprozesse in der Identitätsforschung. In H. Keupp \& R. Höfer (Hrsg.), Identitätsarbeit heute. Klassische und aktuelle Perspektiven der Identitätsforschung (S. 11-39). Frankfurt a. M.: Suhrkamp.

Keupp, H., \& Dill, H. (2010). Vorwort: Erschöpfende Arbeit - Gesundheit und Prävention in der flexiblen Arbeitswelt. In H. Keupp \& H. Dill (Hrsg.), Erschöpfende Arbeit. Gesundheit und Prävention in der flexiblen Arbeitswelt (S. 7-17). Bielefeld: transscript.

Keupp, H., \& Höfer, R. (Hrsg.). (1997). Identitätsarbeit heute. Klassische und aktuelle Perspektiven der Identitätsforschung. Frankfurt a. M.: Suhrkamp.

Krainz, U. (2020). Jenseits des Marktprinzips. Über den politischen Sinn gruppendynamischen Lernens. Gruppe. Interaktion. Organisation. (GIO), 4(51), 489-498. 
Lewin, K. (1947). Frontiers in Group Dynamics. Human Relations, 1(1947), 5-41.

Löw, M. (2019). Raumsoziologie (10. Aufl.). Frankfurt a. M.: Suhrkamp.

March, J., \& Olsen, J. (1975). The uncertainty of the past: organizational learning under ambiguity. European Journal of Political Research, 3(1975), 147-171.

Marr, R. (Hrsg.). (2002). Kaderschmiede Bundeswehr? Vom Offizier zum Manager. Karriereperspektiven von Absolventen der Universitäten der Bundeswehr in Wirtschaft und Verwaltung (2. Aufl.). Neubiberg: gfw.

Oestereich, B., \& Schröder, C. (2020). Agile Organisationsentwicklung. Handbuch zum Aufbau anpassungsfähiger Organisationen. München: Vahlen.

Peters, S. (2012). Projektorganisation und Projektmanagement unter Bedingungen zunehmender Komplexität. In F. Böhle \& S. Busch (Hrsg.), Management von Ungewissheit. Neue Ansätze jenseits von Kontrolle und Ohnmacht (S. 137-176). Bielefeld: transcript.

Peters, S., \& Dengler, S. (2010). Wissenspromotion als Element von Wissensarbeit. In M. Moldaschl \& N. Stehr (Hrsg.), Wissensökonomie und Innovation. Beiträge zur Ökonomie der Wissensgesellschaft (S. 563-588). Marburg: Metropolis.

Peters, S., v. Garrel, J., Düben, A., \& Dienel, H.-L. (2016). Arbeit Zeit - Souveränität. Eine empirische Untersuchung zur selbstbestimmten Projektarbeit (2. Aufl.). München: Hampp.

Peters, S., v. Garrel, J., Düben, A., Dienel, E., \& Dienel, H.-L. (2021). Neue Formen von Arbeitszeitselbstbestimmung in digitalisierten Projektwelten, Abschlussbericht. Berlin: TU Berlin/Nexus Institut.

Reinhardt, K. (2020). Digitale Transformation der Organisation. Grundlagen, Praktiken und Praxisbeispiele der digitalen Unternehmensentwicklung. Berlin: Springer.

Sackmann, S. (Hrsg.). (1997). Cultural complexity in organizations. Inherent contrasts and contradictions. Thousand Oaks: SAGE.

Salazar, Y., \& Peters, S. (2011). Betriebliches Lernen in Händen neuer Akteure - Herausforderungen für die Innovationsfähigkeit von Unternehmen. In S. Jeschke, I. Isenhardt, F. Hees \& S. Trantow (Hrsg.), Enabling Innovation. Innovationsfähigkeit - deutsche und internationale Perspektiven (S. 127-138). Berlin: Springer.

Schmitz, R. (2020). Kollaboratives Arbeiten trotz räumlicher Trennung. Wissensmanagement: das Magazin für Digitalisierung, Vernetzung und Collaboration, 5(22), 26-27.

Schnaufer, H.-G., Stieler-Lorenz, B., \& Peters, S. (2014). Wissen vernetzen. Wissensmanagement in der Produktentwicklung. Heidelberg: Springer.

Schoper, Y., Huemann, M. \& Reschwamm, K. (Hrsg) (2020). Emotionen im Projektmanagement, ProjektmanagementAktuell, 6(31), $4-6$.

Seifert, J. (2005). Cognitive map, Mnemo-Technik und Mind Mapping. Raumeindrücke mental verorten, Wissensstrukturen visualisieren, Vorstellungsräume zum Lernen nutzen. ALFA-FORUM. Zeitschrift für Alphabetisierung und Grundbildung, 60, 32-34.

Tainter, J. (1990). The collapse of complex societies. Cambridge: UP.
Tajfel, H., \& Turner, J. (1986). The social identity theory of intergroup behavior. In S. Worchel \& W. Austin (Hrsg.), Psychology of intergroup relations (2. Aufl. S. 7-24). Chicago: Nelson-Hall.

Tholl, M. (2021): Im digitalen Spiegelkabinett. Der Tagesspiegel am Sonntag. Nr. 24.497 vom 04.04.2021, 5.

Wagner, R., \& Rieteke, S. (Hrsg.). (2014). Theory meets practice in projects. Nürnberg: Gesellschaft für Projektmanagement.

Weick, K., \& Bougon, M. (1986). Organizations as cognitive maps. In H. Sims \& G. Dennis (Hrsg.), The thinking organization: dynamics of organizational social cognition (S. 102-135). San Francisco: Jossey-Bass.

Wirth, K. (2020). Verwaltung agiler machen! Hype oder kalter Kaffee? Verwaltung \& Management (VM), 4, 161-168.

Wust, P. (1940). Ungewissheit und Wagnis (3. Aufl.). München: KöselPustet.

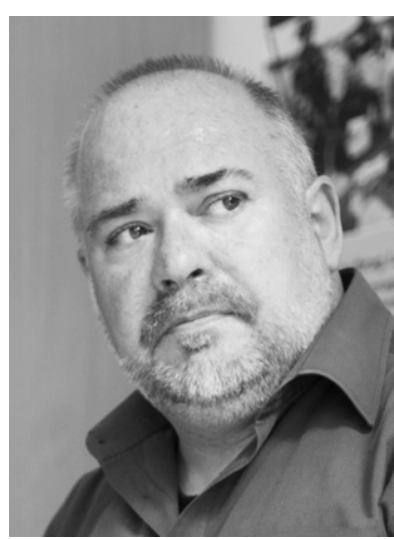

Prof. Dr. Martin Elbe forscht am Zentrum für Militärgeschichte und Sozialwissenschaften der Bundeswehr und lehrte als Professor für Arbeits- und Organisationspsychologie an verschiedenen Hochschulen. Seine Arbeitsschwerpunkte liegen im Bereich der Sozialpsychologie und Militärsoziologie (insbesondere: Organisation, Arbeit, Personal, Gesundheit, Sport, Verstehen).

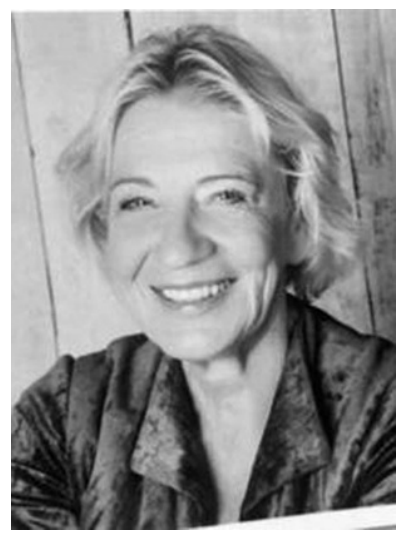

Prof. Dr. Sibylle Peters ist als Gastwissenschaftlerin im Bereich Arbeitslehre/Technik und Partizipation der TU Berlin tätig und war bis 2011 Professorin für betriebliche Weiterbildung und Personalentwicklung an der Universität Magdeburg. Ihre aktuellen Arbeitsschwerpunkte liegen im Bereich Führungsnachwuchs, Arbeitszeitsouveränität, Wissens- und Projektmanagement. 